\title{
Establishment and characterization of BHD-F59RSVT, an immortalized cell line derived from a renal cell carcinoma in a patient with Birt-Hogg-Dubé syndrome
}

\author{
Mitsuko Furuya', Hisashi Hasumi ${ }^{2}$, Masaya Baba ${ }^{3}$, Reiko Tanaka ${ }^{4}$, Yasuhiro Iribe ${ }^{1,2}$, Takahiro Onishi ${ }^{5}$, Yoji Nagashima ${ }^{6}$, \\ Yukio Nakatani ${ }^{7}$, Yasuhiro Isono ${ }^{8}$ and Masahiro Yao $^{2}$
}

Hereditary renal cell carcinomas (RCCs) are life-threatening disorders not only for the patients but also for their relatives. Birt-Hogg-Dubé syndrome (BHD) is an autosomal dominant disorder caused by germline mutations in the folliculin gene $(F L C N)$. The protein product, FLCN, functions as a tumor suppressor, and the affected patients have high risks of developing multiple RCCs. The carcinogenic mechanisms stemming from FLCN dysfunction have been investigated using rodent models and human RCC tissues. However, very limited information has been available about in vitro signaling of human renal cells with genetically mutant FLCN. Herein, we established a new cell line, BHD-F59RSVT, from a BHD patient's chromophobe RCC by transfecting SV40 large T antigen. We investigated FLCN mutations, chromosome profiles, and cytopathologic characteristics of the cell line. BHD-F59RSVT reflected the patient's FLCN germline mutation, a 3-nt deletion in exon 13 (c.1528_1530delGAG). Neither somatic mutation nor loss of heterozygosity of FLCN was detectable.

Chromosome 17p11.2 of the FLCN proximal region demonstrated a trimodal pattern. Genome-wide chromosomal analysis revealed a loss of chromosome 16 and mosaic segmental gains in chromosome 7. BHD-F59RSVT cells were positive when immunostained for cytokeratin 7, supporting their origin from distal convoluted tubules. Western blotting analysis demonstrated severely suppressed FLCN expression at the protein level. The collective findings indicate that the established cell line will be suitable for functional analysis of the typical phenotype of BHD-associated RCC with suppressed FLCN expression.

Laboratory Investigation (2017) 97, 343-351; doi:10.1038/labinvest.2016.137; published online 19 December 2016

Hereditary renal cell carcinomas (RCCs) require special management so that patients and their families can avoid dialysis and progression to cancer-related death. Birt-HoggDubé syndrome (BHD), also called Hornstein-Knickenberg syndrome, is an inherited disorder characterized by skin fibrofolliculomas, multiple pulmonary cysts, and renal tumors. ${ }^{1,2}$ The responsible gene for BHD, folliculin (FLCN), is located at $17 \mathrm{p} 11.2,{ }^{3}$ and its protein product, FLCN, forms a complex with interacting partners FNIP1 and FNIP2. ${ }^{4-6}$ The principal role of FLCN in human diseases is tumor suppression, and the mechanism involves several pathways, including mammalian target of rapamycin and 5'-AMPactivated protein kinase. ${ }^{4}$ It is known that a variety of renal tumor subtypes, including clear cell, papillary, chromophobe, and oncocytoma, may develop in BHD patients, although chromophobe RCC and hybrid oncocytic/chromophobe tumors (HOCTs) are predominant. ${ }^{7,8}$

Detailed mechanisms of FLCN function in the kidney have been intensively studied using mice with Flcn inactivation in epithelial cells of the kidney, ${ }^{9,10}$ or immortalized human kidney epithelial cells treated with FLCN siRNA. ${ }^{11,12}$ Only one cell line, UOK257, from a BHD patient's RCC is currently used for functional studies of FLCN in vitro. UOK257 was established from a high-grade RCC characterized as predominantly clear cell type with aggressive behavior. ${ }^{13}$ The majority of renal tumors developing in BHD patients are

\footnotetext{
Department of Molecular Pathology, Yokohama City University Graduate School of Medicine, Yokohama, Japan; ${ }^{2}$ Department of Urology, Yokohama City University Graduate School of Medicine, Yokohama, Japan; ${ }^{3}$ International Research Center for Medical Sciences (IRCMS), Kumamoto University, Kumamoto, Japan; ${ }^{4}$ Medical Mycology Research Center, Chiba University, Chiba, Japan; ${ }^{5}$ Department of Internal Medicine, Ise Red Cross Hospital, Ise, Japan; ${ }^{6}$ Department of Surgical Pathology, Tokyo Women's Medical University, Tokyo, Japan; 'Department of Diagnostic Pathology, Chiba University Graduate School of Medicine, Chiba, Japan and ${ }^{8}$ Department of Otolaryngology, Yokohama City University Graduate School of Medicine, Yokohama, Japan

Correspondence: Dr M Furuya, MD, PhD, Department of Molecular Pathology, Yokohama City University Graduate School of Medicine, 3-9 Fukuura, Kanazawa-ku, Yokohama 236-0004, Japan.

E-mail:mfuruya@yokohama-cu.ac.jp

Received 17 May 2016; revised 16 October 2016; accepted 17 November 2016
} 


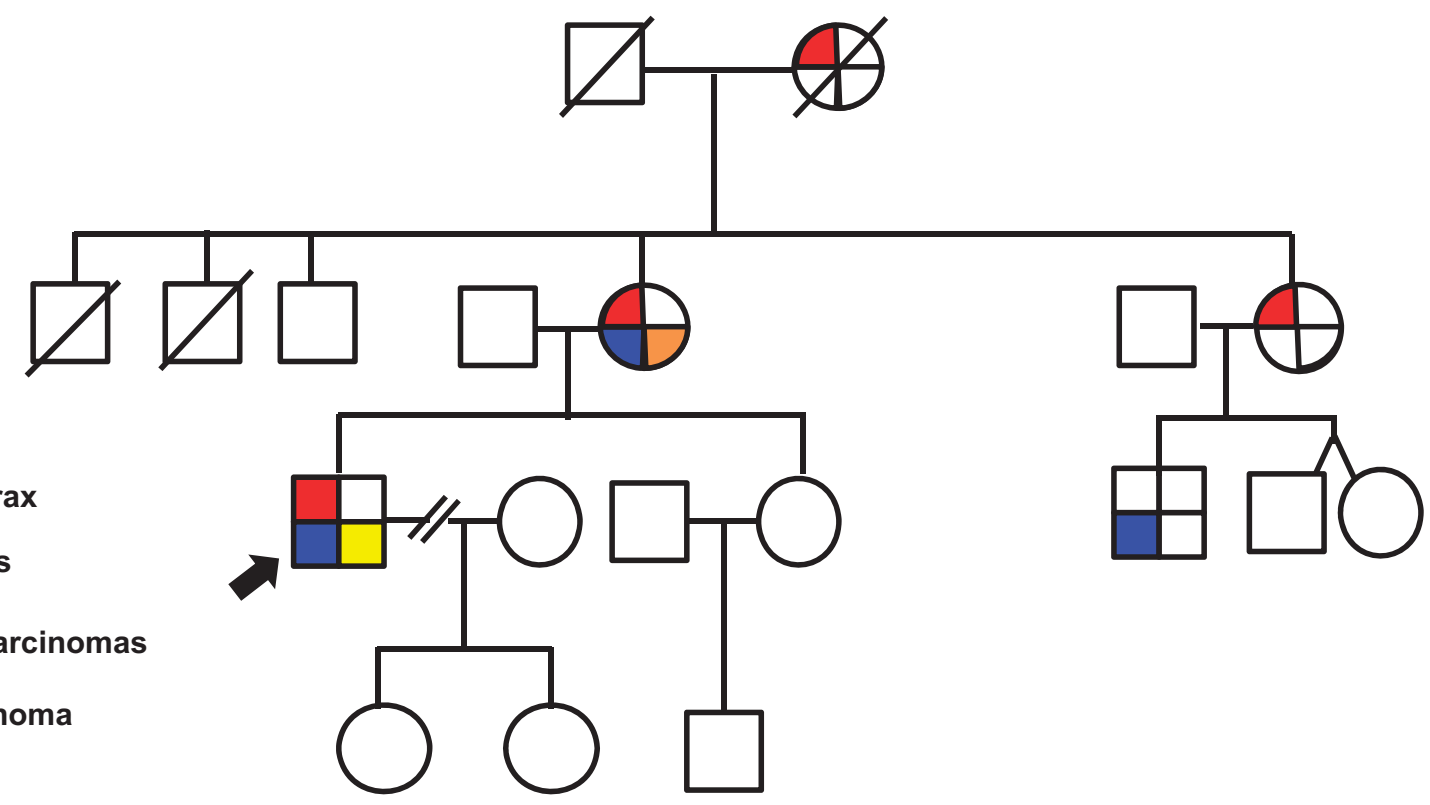

b

Pneumothorax

Skin papules

Renal cell carcinomas

Colon carcinoma
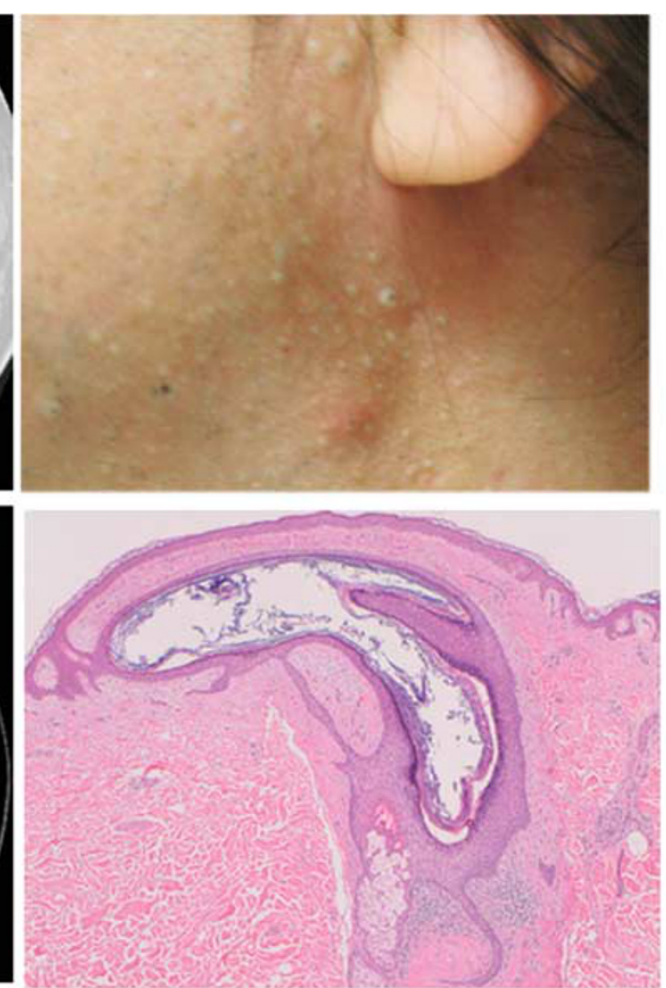

Germline mutation

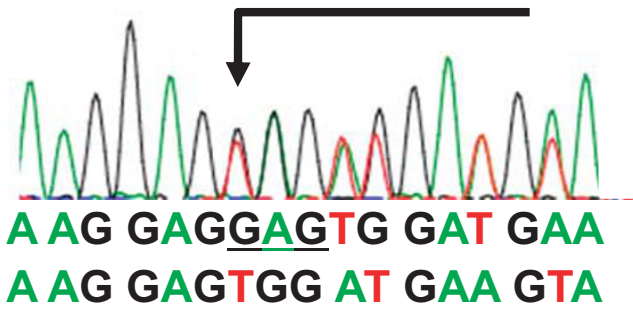

c.1528_30delGAG 
chromophobe RCC and HOCT, and these tumor subtypes tend to be less aggressive in behavior. ${ }^{14-16}$ Herein we established an immortalized cell line derived from a chromophobe RCC in a Japanese BHD patient. We characterized the clinicopathologic information, germline mutation, and cytogenetic features of the established cell line.

\section{MATERIALS AND METHODS}

\section{Cell Culture Information}

A patient with multiple RCCs received genetic counseling and agreed to undergo genetic testing. Written informed consent was obtained from the patient for cytogenetic and clinicopathologic analyses. The study was approved by the Institutional Review Board (IRB) of Yokohama City University. A part of the resected tumor was fixed with $10 \%$ buffered formalin and embedded in paraffin. Hematoxylin and eosin (HE) staining was performed for histological diagnosis. The other parts of the tumor were either used for cell culture or snap-frozen and stored in liquid nitrogen until cytogenetic analysis.

The tumor tissue was minced with a sterile scalpel into 1 -mm-sized fragments, and treated for $30 \mathrm{~min}$ with $1 \mathrm{mg} / \mathrm{ml}$ collagenase (Wako Pure Chemical Industries, Osaka, Japan). The cells were rinsed and cultured in RPMI1640 (Life Technologies, Carlsbad, CA, USA) with $10 \%$ heatinactivated fetal bovine serum, and $1 \%$ penicillin and streptomycin. One week after plating, adherent epithelial cell overgrowth was observed. These epithelial cells (passage one) were treated with $0.05 \%$ trypsin-EDTA and then cultured in 12 -well plates at $8 \times 10^{4}$ cells per well. The cells were infected with SV40 large $\mathrm{T}$ antigen (SV40gp6)-expressing lentiviral vector pLSVSIN-EF1a (Takara Bio, Shiga, Japan) according to the manufacturers' instructions. The cells were incubated with the plasmid DNA for $24 \mathrm{~h}$ and were then washed, and transferred to $10-\mathrm{cm}$ dishes. When the dishes were subconfluent, the cells were diluted for cloning. After puromycin selection, immortalized SV40gp6-expressing cells was obtained and named BHD-F59RSVT. The expression of SV40gp6 was then confirmed by RT-PCR. BHD-F59RSVT cells were cultured in Renal Epithelial Cell Growth Medium 2 with recombinant human epithelial growth factor (PromoCell $\mathrm{GmbH}$, Heidelberg, Germany), and $1 \%$ penicillin and streptomycin. The cells were stepwisely cultured from 96to 6-well plates, then proliferated in $25-\mathrm{cm}^{2}$ flasks. Further analysis was performed using one to two passage cells of stably established cells.

\section{Antibodies}

For western blotting of FLCN, rabbit monoclonal antibody D14G9 (Cell Signaling, Danvers, MA, USA) was used. Mouse monoclonal antibody against $\beta$-actin was purchased from Sigma (St Louis, MO, USA). For cytostaining and histopathologic studies, we used rabbit polyclonal antibodies against FLCN (ab93196, Abcam, Cambridge, UK) and smooth muscle actin (SMA; ab5694, Abcam). We also utilized mouse monoclonal antibodies against cytokeratin 7 (CK7; Dako, Glostrup, Denmark), CD82 (Santa Cruz Biotechnology, Santa Cruz, CA, USA), mitochondria (Clone 113-1, BioGenex, Fremont, CA, USA), CD10 (Dako), RCC (Dako), and cytokeratin AE1/AE3 (Dako).

\section{Immunofluorescence Cytostaining}

BHD-F59RSVT cells were seeded on chamber slides, and $24 \mathrm{~h}$ later, the slides were washed with PBS, fixed with $4 \%$ paraformaldehyde for $10 \mathrm{~min}$, and permeabilized with $0.5 \%$ Triton X-100. After washing three times with PBS and blocking with $5 \%$ blocking reagent for $30 \mathrm{~min}$, cells were labeled with primary antibodies for $90 \mathrm{~min}$, and then incubated with Alexa Fluor 488 goat anti-mouse $\operatorname{IgG}$ or anti-rabbit IgG (Life Technologies) for $30 \mathrm{~min}$ at room temperature. Working dilutions were 1:50 for FLCN and mitochondria and 1:100 for other antibodies. UOK257-2 was used for FLCN-positive control. This cell line restores wildtype FLCN. ${ }^{11}$

\section{Immunohistochemistry}

Four- $\mu \mathrm{m}$-thick paraffin sections of the original tumor and BHD-F59RSVT cell pellets were subjected to immunohistochemistry. After deparaffinization and rehydration, sections were autoclaved at $121^{\circ} \mathrm{C}$ for $15 \mathrm{~min}$, after which they were treated with the diluted antibodies at $4{ }^{\circ} \mathrm{C}$ overnight. Immunohistochemical staining was done using the EnVision ${ }^{+}$ kit (Dako), followed by 3, 3'-diaminobenzidne staining for visualization. Working dilutions were 1:50 for FLCN and CK7, and 1:100 for other antibodies. ${ }^{16}$

\section{DNA Isolation}

DNA from peripheral blood leukocytes was obtained using the LabPass Blood Mini kit (Cosmo GENETECH, Seoul, Korea). DNA from renal tissue was obtained using a QIAamp DNA Mini kit (Qiagen, Hidden, Germany) according to the manufacturers' instructions.

Figure 1 Family tree, symptoms, and FLCN germline mutation of a patient with renal tumors. (a) The patient is indicated by an arrow. Four members including the proband recognized skin papules (in red). The proband underwent skin biopsy and FLCN genetic testing. The other members refused to undergo diagnostic workup. (b) Computed tomography shows multiple pulmonary cysts (indicated by arrows in upper left) and renal tumors (indicated by arrow heads in lower left). The patient had multiple small dome-shaped skin tumors (upper right) that were histologically diagnosed as fibrofolliculomas (lower right). (c) Direct sequencing of FLCN exon 13. Control normal sequencing (left) and sequencing of the patient's blood cell DNA (right) are shown. The underlined nucleotides (c.1528_30delGAG) are heterozygously deleted (c.1528 is indicated by an arrow). FLCN, folliculin. 

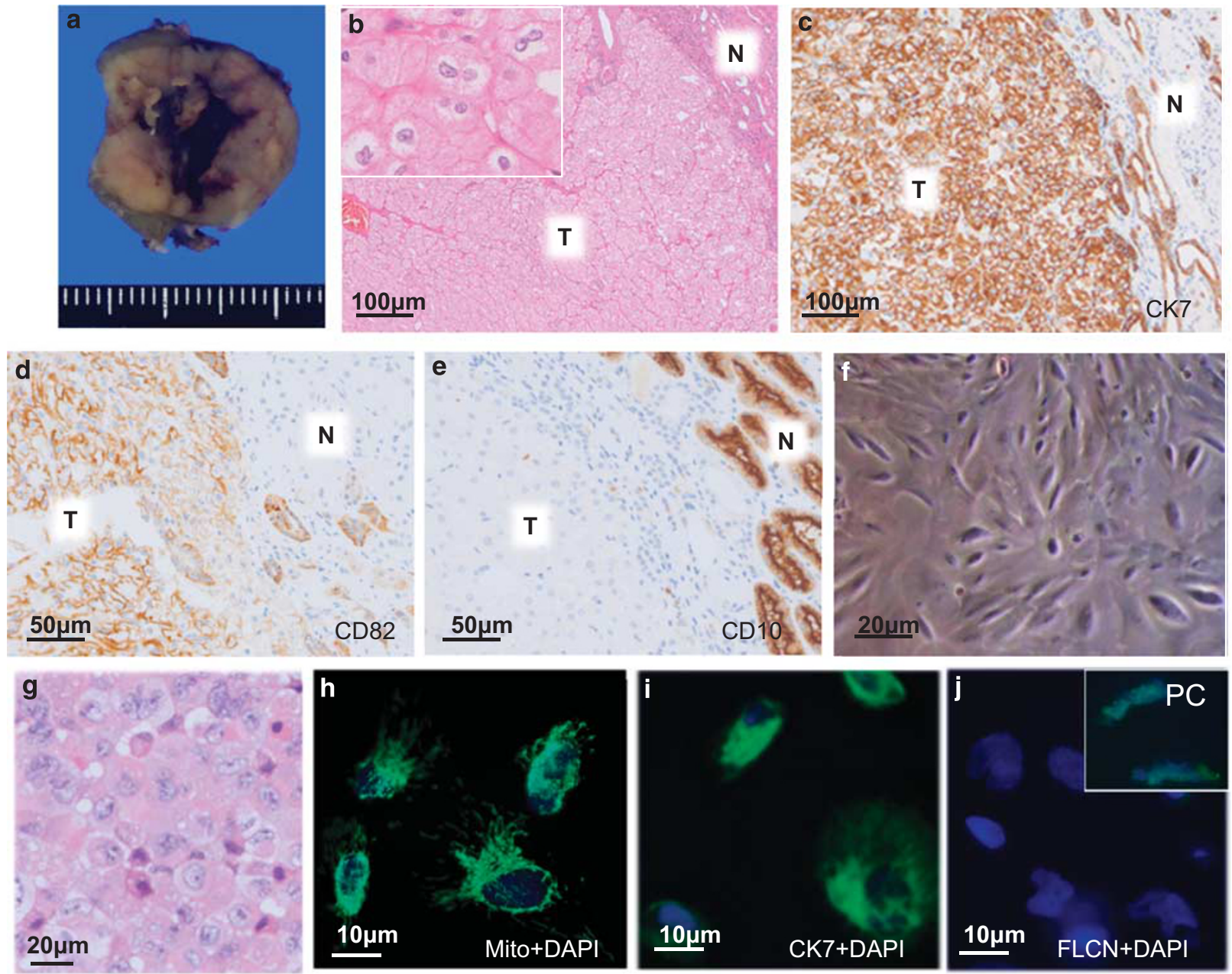

$\mathbf{k}$

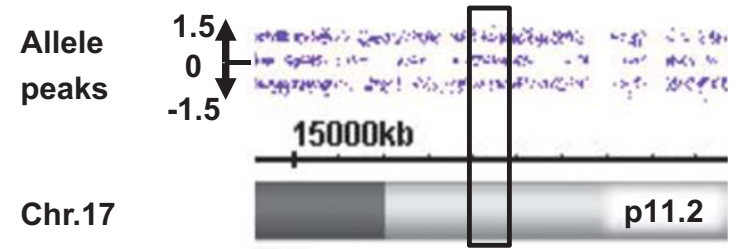

$17116 \mathrm{~Kb}-17145 \mathrm{~Kb}$

(FLCN-proximal segment)
I

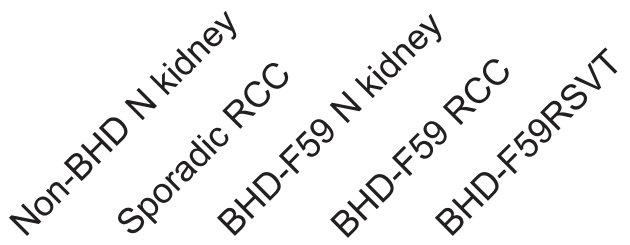

FLCN

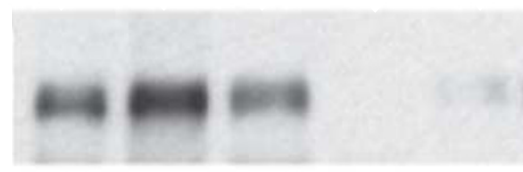

$\beta$-actin

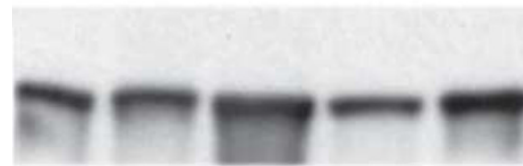

\section{Direct Sequencing and PCR}

Exons 1-14 of the FLCN gene were amplified by PCR using the primers described previously. ${ }^{3}$ In addition, the copy number of each exon was assessed using quantitative PCR to detect large rearrangements. ${ }^{17}$ PCR conditions were described in our previous study. ${ }^{18}$ After purification, DNA was labeled with a Big Dye Terminator v1.1 Cycle Sequencing Kit (Applied Biosystems, Cleveland, OH, USA) and DNA sequencing was done using an ABI Prism 3100 Genetic Analyzer (Applied Biosystems). 
Figure 2 Morphologies of the original renal tumor and the immortalized cell line. (a) The cut surface of the tumor was brownish in color with central bleeding. (b) Hematoxylin and eosin staining of the tumor. The tumor (T) was composed of eosinophilic and granular cells with perinuclear halos. Raisinoid nuclei and binucleation were observed (inset). Non-tumor area was indicated as N. (c-e) The tumor (T) was diffusely immunostained for cytokeratin 7 (CK7, in c), CD82 (d), and negatively stained for CD10 (e). Non-tumor area was indicated as N. Normal distal tubules were stained for CK7 and CD82, and normal proximal tubules were stained for CD10. (f) Morphology of BHD-F59RSVT in vitro. The cells were ovoid in shape, and some have foot processes. (g) Hematoxylin and eosin staining of BHD-F59RSVT cell pellets showed granular cytoplasm. (h-j) Fluorescent immunostainings of BHDF59RSVT. The cells were diffusely immunostained for mitochondria (Mito, in h) and CK7 (i), but scarcely stained for FLCN (j). Inset: positive control (PC) of FLCN cytostaining in UOK257-2 cells. (k) Chromosome 17 analysis of BHD-F59RSVT. The FLCN proximal region in p11.2 exhibited a normal trimodal pattern. Neither gain/loss nor loss of heterozygosity was detected. (I) Western blotting of FLCN. N indicated a normal region and RCC indicated a tumor region. The FLCN bands ( $64 \mathrm{kDa}$ ) were clearly detected in non-tumor lanes and a sporadic chromophobe RCC, but barely seen in the original BHD-F59 RCC (homogenized tissue) and BHD-F59RSVT. BHD, Birt-Hogg-Dubé syndrome; FLCN, folliculin; RCC, renal cell carcinoma.

\section{Methylation Analysis}

The promoter status of FLCN was examined by a methylation-specific PCR approach using a CpGenome Turbo Bisulfate Modification kit (Merck Millipore, Darmstadt, Germany). DNA was treated with sodium bisulfate according to the manufacturers' instructions. The specific primers and PCR conditions were described previously. ${ }^{19}$ The PCR product was treated with $R s a \mathrm{I}$ for $2 \mathrm{~h}$ at $37^{\circ} \mathrm{C}$. The restriction enzyme digestion product was then visualized in $2 \%$ agarose gel.

\section{Western Blotting}

Lysates (12.5 $\mu \mathrm{g}$ protein) were electrophoresed on SDS-PAGE and transferred to a PVDF membrane (Millipore, Billerica, MA, USA). Horseradish peroxidase-conjugated goat antirabbit IgG and anti-mouse IgG (Santa Cruz Biotechnology) were used as the secondary antibodies. Bands were detected using an enhanced chemiluminescence system, according to the Hybond ECL protocol (GE Healthcare, Buckinghamshire, UK).

\section{RNA Isolation and RT-PCR}

Total RNAs were isolated from cells using TRIzol Reagents and reverse transcription was done using SuperScript III according to the manufacturer's protocols (Life Technologies). The primers used for PCR were as follows: SV40gp6, (F) 5'-GGCTACTGCTGACTCTCAACATTCT-3' and (R) 5'-AG CAGAGCCTGTAGAA CCAAACATT-3'; GAPDH (F) 5'-AAG GTGAAGGTCGGAGTCAAC-3' and (R) 5'-CATGAGTCCTT CCACGATACC-3'. The expression levels of FLCN mRNA were investigated according to the method previously described. ${ }^{11}$

CytoScan HD Array and Copy-Number Variation Analysis A CytoScan HD Array (Affymetrix, Santa Clara, CA, USA) was used to analyze genomic alterations according to the manufacturer's protocol. In brief, $250 \mathrm{ng}$ of genomic DNA from BHD-F59RSVT was digested with the restriction enzyme Nsp I and then ligated to an adapter, followed by PCR amplification. The data were analyzed using Affymetrix Chromosome Analysis Suite (ChAS) version 3 to detect copynumber variation $(\mathrm{CNV})$ and loss of heterozygosity $(\mathrm{LOH})$.
Definitions of $\mathrm{CNV}$ and $\mathrm{LOH}$ were presented in our previous study. ${ }^{20}$ The allelic patterns were interpreted as follows. A normal $\log _{2}$ ratio with trimodal allele peaks indicated biparental disomy (normal). A normal $\log _{2}$ ratio with bimodal allele peaks indicated uniparental disomy (UPD). An increased $\log _{2}$ ratio with tetramodal allele peaks indicated trisomy. A decreased $\log _{2}$ ratio with bimodal allele peaks indicated monosomy. A decreased $\log _{2}$ ratio with tetramodal allele peaks indicated mosaic loss.

\section{Spectral Karyotyping Analysis}

Spectral karyotyping (SKY) analysis was performed to characterize aberrant chromosome using the 24-color SKY probe system (Applied Spectral Imaging (ASI), Carlsbad, CA, USA). The chromosome preparations were hybridized with the SKY probe mixture for $48 \mathrm{~h}$ at $37^{\circ} \mathrm{C}$. The multicolor hybridization was visualized by Spectra Cube (ASI) and images were analyzed using SKYVIEW software (ASI). Five cell lines were analyzed. Chromosomal abnormalities were described according to an International System for Human Cytogenetic Nomenclature (ISCN) 2013. If two or more cells contained an identical structural aberration, the aberrations were considered recurring, and if three or more cells demonstrated an identical chromosomal loss, the loss was considered clonal.

\section{RESULTS \\ Clinicopathologic Manifestations and Germline FLCN Mutation}

A 52-year-old Japanese man with repeated episodes of pneumothorax was admitted to our hospital for resection of multiple renal tumors. He had experienced skin papules in adolescence, and papules developed in the arms, legs, head, neck, and trunk. His mother and a cousin had episodes of pneumothorax. Although his mother, an aunt, and a grandmother also reported subtle skin papules, none consulted dermatologists (Figure 1a). His mother underwent colon cancer surgery at the age of 72 , but no neoplasm was detected in the kidney. Computed tomography of the proband revealed multiple pulmonary cysts and bilateral renal tumors (Figure 1b). His medical record, together with a family history of pneumothorax, alerted us to the possibility of BHD. 
a

Chromosome

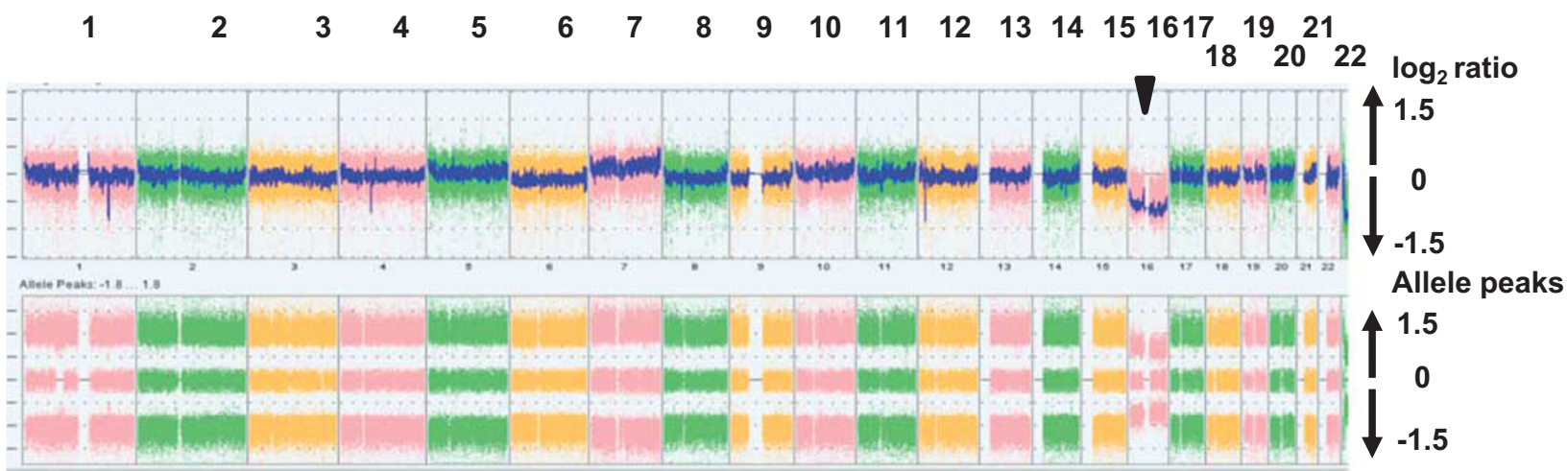

b

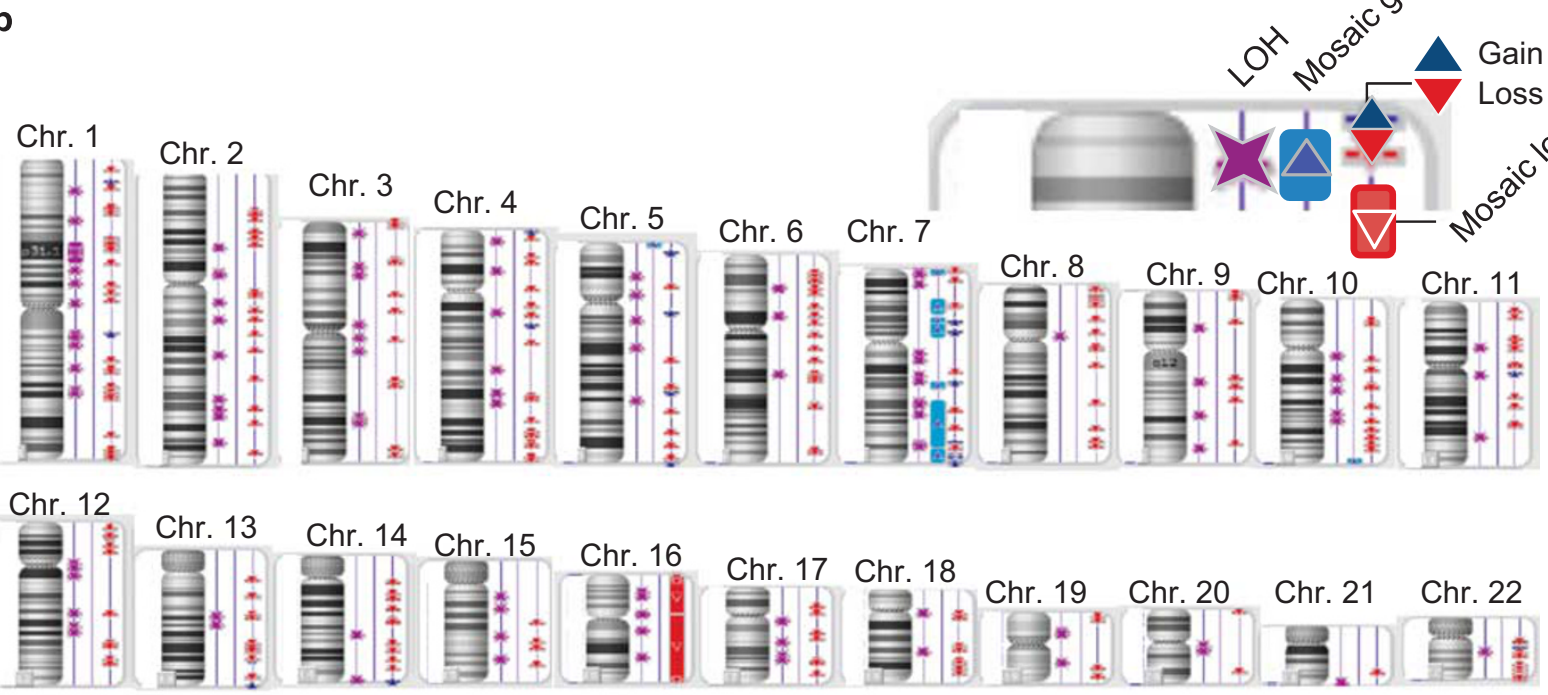

C

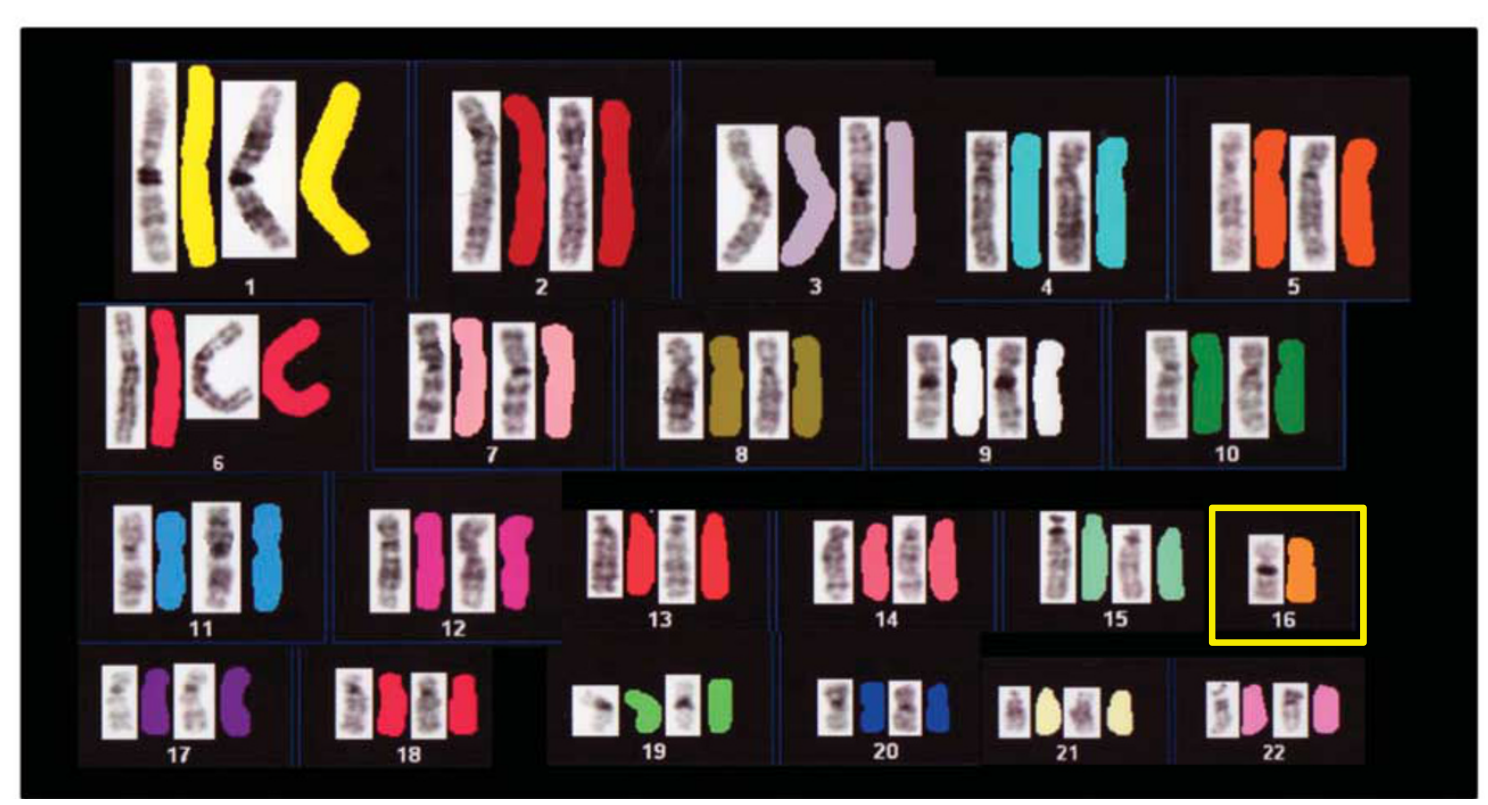


Figure 3 Broad chromosomal analysis, loss of heterozygosity (LOH), and spectral karyotyping (SKY) for BHD-F59RSVT. (a) Copy-number ratios (log 2 ratios (top panels)) and allele peaks (bottom panels) of chromosomes 1-22 (the left end, chromosome 1; the right end, chromosome 22) are shown. Chromosome 16 shows a decreased $\log _{2}$ ratio (arrowhead); chromosome 7 shows a slight gain although $\ln \log _{2}$ ratio is within the balanced copy number. (b) LOH and gains/losses of BHD-F56RSVT detected by CytoScan HD Array are shown. Purple stars indicate LOH. Blue and red triangles indicate segmental gains and losses, respectively. (c) The SKY data are presented for chromosomes 1-22 (the left upper, chromosome 1; the right bottom, chromosome 22). Inverted DAPI-stained chromosomes are at the left, and pseudo-colored chromosomes hybridized with SKY probes are at the right. SKY of chromosome 16 is enclosed with a yellow rectangle.

Genetic counseling was performed, and informed consent was obtained from the patient for FLCN genetic testing after approval of IRB. A 3-bp deletion in exon 13 (c.1528_30delGAG) was detected (Figure 1c). The mutation was reported by Toro et al as pathogenic, predicting amino-acid change $\mathrm{p}$. Glu510del. ${ }^{2}$ The patient was diagnosed with BHD. His mother and siblings were not willing the genetic testing.

$\mathrm{He}$ underwent asynchronous bilateral nephron-sparing surgeries, and multiple tumors were resected (seven from the left and four from the right). All tumors exhibited oncocytic cytoplasm with perinuclear halo, and they were histologically diagnosed as HOCTs $(n=3)$ and chromophobe RCCs $(n=8)$. A few biopsied skin papules were histologically diagnosed as fibrofolliculomas. The patient recovered without complication, and receives periodic medical check-up.

\section{Cytology and FLCN Status of BHD-F59RSVT}

An immortalized cell line BHD-F59RSVT was established from one of the resected chromophobe RCCs. The tumor was obtained from the patient's right kidney, a $12 \times 14$-mm-sized nodule with central bleeding. The tumor cells had granular or oncocytic cytoplasms, and round to raisinoid nuclei with perinuclear halos. Tumor cells were positively immunostained for CK7 and CD82, but they were negative for CD10 and RCC marker (Figures 2a-e). These immunostaining patterns indicated that the tumors were derived from distal tubules or collecting ducts.

The BHD-F59RSVT cells were plump or oval in shape. HE and cytostaining showed eosinophilic granular cytoplasm, supporting their oncocytic nature. Cytostaining analysis demonstrated that the cells expressed CK7, mitochondrial antigen, and cytokeratin AE1/AE3, but were negative for SMA, supporting the interpretation that these oncocytic epithelial cells were derived from distal tubules (Figures $2 \mathrm{~d}-\mathrm{g})$. The cells were scarcely immunostained for FLCN (Figure 2h). Sequence analysis of exons 1-14 and donor/ acceptor sites of introns was performed to clarify somatic second hit mutations of FLCN. BHD-F59RSVT demonstrated neither small intragenic mutations nor LOH of FLCN. The cells exhibited the same mutation pattern (c.1528_30delGAG) as that in the germ line (Supplementary Figure S1). Quantitative PCR analysis of exons 1-14 argued against potential deletion/duplication in the FLCN gene (data not shown). Further cytogenetic analysis of FLCN proximal regions in chromosome $17 \mathrm{p} 11.2$ demonstrated normal allele peaks and a trimodal pattern (Figure 2i). Methylation was not detectable in FLCN (data not shown); however, western blot analysis revealed that FLCN expression at the protein level was severely suppressed in BHD-F59RSVT as well as in the original RCC tissue (Figure 2j). Quantitative RT-PCR demonstrated suppressed FLCN mRNA in BHD-F59RSVT and original tumor compared with those in sporadic RCCs. Significant difference was not observed between BHDF59RSVT and normal kidneys in which FLCN was preserved at the protein level (Supplementary Figure S2).

\section{Chromosomal Analysis and CNV}

Chromosomal analysis of BHD-F59RSVT revealed a loss of chromosome 16 and slight mosaic gains of chromosome 7 (Figure 3a). Other chromosomes demonstrated balanced chromosomal copy numbers. The pattern was distinctively different from that of sporadic chromophobe RCC characterized by the losses of chromosomes $1,2,6,10,13,17$, and $21{ }^{21}$

$\mathrm{CNV}$ analysis demonstrated numerous segmental gains, losses, and LOHs distributed over all chromosomes (Figure 3b; Supplementary Figure S3). Mosaic losses were consistently detected throughout in chromosome 16, and mosaic gains were detected in chromosome 7 . There were 66 copy-number gains (2861 kbp in sum) and 9 mosaic gains (96059 kbp in sum), and 294 copy-number losses (93 $952 \mathrm{kbp}$ in sum. It was not possible to distinguish between losses and mosaic losses because of low-level mosaic detection). Each chromosome had at least one LOH lesion. There were $105 \mathrm{LOHs}$ (305 $306 \mathrm{kbp}$ in sum). In chromosome 16, LOH lesions showed either monosomy or chromosomal loss. In other chromosomes, almost all regions were copyneutral LOH, that is, UPD. In SKY analysis, allelic loss of chromosome 16 was detected in three of five cells (Figure 3c), which was considered to be clonal according to ISCN. Other abnormalities were as follows: allelic loss of chromosome 22 was in one cell, and a translocation involving chromosome 7 in 2 of 5 cells.

We also examined chromosomal state of the original tumor for comparison. The tumor demonstrated balanced copy numbers including chromosome 16 (Supplementary Figure S4A). The $\mathrm{LOH}$ and gain/loss patterns were almost same to those in BHD-F59RSVT (Supplementary Figure S4B). In the original tumor, regional LOHs of chromosome 7 and 16 were almost identical to those in BHD-F59RSVT, but mosaic gains/losses were not detected. SKY analysis using the primary culture cells of the original tumor was performed. One of five cells demonstrated deletion 
of the short arm of chromosome 16 (Supplementary Figure S4C). Collectively, it was suggested that BHDF59RSVT lost $16 \mathrm{p}$ during clonal selection, leading to an allelic loss of chromosome 16 in the immortalization process.

\section{DISCUSSION}

We established a new cell line that carries a mutant FLCN gene. It originated from a chromophobe RCC in a patient with BHD. The cell line, as well as the original tumor tissue, had neither a second hit nor promotor methylation of FLCN; however, its protein product (FLCN) was severely suppressed. It is assumed that some unknown mechanisms suppressed FLCN production both in vivo and in vitro. It is apparent that a second hit of FLCN may not be essential for FLCN suppression. BHD-F59RSVT had granular eosinophilic cytoplasm and demonstrated positive immunostaining for mitochondria. These morphologic characteristics were similar to those of the original RCC. BHD-F59RSVT may be a suitable model for investigating molecular pathways in the presence of impaired FLCN.

BHD-F59RSVT was established from an RCC with a typical chromophobe phenotype. Thus, this cell line is expected to reflect the nature of RCCs occurring in the majority of BHD patients. As Klomp et al and our group described previously, BHD-associated oncocytic RCCs have different chromosomal characteristics from those in sporadic chromophobe RCCs. ${ }^{20,22,23}$ BHD-F59RSVT exhibited balanced state of chromosomes 2, 6, 10, 13, 17, and 21. Frequent losses of these chromosomes are observed in sporadic chromophobe RCCs. $^{21,24}$ The unique features of BHD-F59RSVT may contribute to clarification of the distinct carcinogenic mechanism in which impaired FLCN is involved. On the other hand, BHD-F59RSVT showed a loss of chromosome 16. This chromosomal alteration during the immortalization process was not seen in previously reported BHD-associated RCCs. ${ }^{20,22}$ More than one RCC-associated gene is on chromosome 16, including TSC2 and PKD1. Deletion of $16 \mathrm{p}$ was detected in one of the primary culture cells. It was likely that a subset of primary culture cells had segmental loss of chromosome 16p during proliferation, leading to an allelic loss in the immortalization process. As FLCN and TSC2 are both negative regulators of mTOR signaling, TSC2 in this cell line might be exposed to continuous stress of accelerated mTOR signaling for cell proliferation and protein synthesis.

There is also a concern about miscellaneous effects associated with transfection. SV40 large T antigen potentially inactivates several tumor suppressor genes including $p 53$, $p R B$, and SEN6. Although chromosome segments containing these genes showed no pathologic event in BHD-F59RSVT, we cannot deny the possibility of small intragenic mutations and/or functional alterations of these factors. At least, we did not detect highly malignant transformation possibly related to SV40 large $\mathrm{T}$ antigen. In our preliminary in vivo experiment using nude mice, inoculated BHD-F59RSVT failed to form a tumor mass in 8 weeks (data not shown). Although longer surveillance is needed to characterize BHD-F59RSVT in vivo, this cell line may reflect less aggressive nature of the patient's tumor that developed slowly without destructive infiltration.

The role of FLCN in the kidney cells is incompletely understood. The kidney-specific Flcn knockout mice develop polycystic kidneys and die due to renal failure. ${ }^{9}$ Cystic change of the kidney is also often observed in patients with BHD. ${ }^{18}$ Therefore, FLCN in renal epithelium appears to be involved not only in carcinogenesis but also in some metabolismassociated morphologic alterations. BHD-F59RSVT has the nature of distal tubules of the kidney, enriched with mitochondria, and impaired expression of FLCN. We hope that BHD-F59RSVT with heterozygous FLCN mutation will contribute to the studies of renal pathology, including renal carcinogenesis, polycystic kidney, and metabolic disorders in which FLCN is possibly involved.

Supplementary Information accompanies the paper on the Laboratory Investigation website (http://www.laboratoryinvestigation.org)

\section{ACKNOWLEDGMENTS}

We thank Ms Hiromi Soeda, Mr Masato Kawashima, and members of the pathology laboratories in Yokohama City University Hospital for excellent assistance. This work was supported by JSPS KAKENHI grant number 26460422 (MF), 26460456 (Yo.N.), 15K08374 (Yu.N.), 15604475 (MB), 15551829 (MB), and 15K10600 (MY), the Mitsubishi Foundation 26329 (MF), Yokohama Foundation for Advancement of Medical Science (MF), Yokohama Academic Foundation (MF), Project Mirai Cancer Research Grants (MF), and Project for Development of Innovative Research on Cancer Therapeutics (P-DIRECT), Ministry of Education, Culture, Sports, Science and Technology of Japan (MY).

\section{DISCLOSURE/CONFLICT OF INTEREST}

The authors declare no conflict of interest.

1. Menko FH, van Steensel MA, Giraud S, et al. Birt-Hogg-Dube syndrome: diagnosis and management. Lancet Oncol 2009;10:1199-1206.

2. Toro JR, Wei MH, Glenn GM, et al. BHD mutations, clinical and molecular genetic investigations of Birt-Hogg-Dube syndrome: a new series of 50 families and a review of published reports. J Med Genet 2008;45:321-331.

3. Nickerson ML, Warren MB, Toro JR, et al. Mutations in a novel gene lead to kidney tumors, lung wall defects, and benign tumors of the hair follicle in patients with the Birt-Hogg-Dube syndrome. Cancer Cell 2002;2:157-164.

4. Baba M, Hong SB, Sharma N, et al. Folliculin encoded by the BHD gene interacts with a binding protein, FNIP1, and AMPK, and is involved in AMPK and mTOR signaling. Proc Natl Acad Sci USA 2006;103: 15552-15557.

5. Hasumi H, Baba M, Hong SB, et al. Identification and characterization of a novel folliculin-interacting protein FNIP2. Gene 2008;415:60-67.

6. Takagi $\mathrm{Y}$, Kobayashi $\mathrm{T}$, Shiono $\mathrm{M}$, et al. Interaction of folliculin (Birt-Hogg-Dube gene product) with a novel Fnip1-like (FnipL/Fnip2) protein. Oncogene 2008;27:5339-5347.

7. Pavlovich $C P$, Walther MM, Eyler RA, et al. Renal tumors in the Birt-Hogg-Dube syndrome. Am J Surg Pathol 2002;26:1542-1552.

8. Srigley JR, Delahunt B, Eble JN, et al. The International Society of Urological Pathology (ISUP) Vancouver Classification of Renal Neoplasia. Am J Surg Pathol 2013;37:1469-1489.

9. Baba M, Furihata M, Hong SB, et al. Kidney-targeted Birt-Hogg-Dube gene inactivation in a mouse model: Erk1/2 and Akt-mTOR activation, cell hyperproliferation, and polycystic kidneys. J Natl Cancer Inst 2008;100:140-154. 
10. Chen J, Futami K, Petillo $D$, et al. Deficiency of FLCN in mouse kidney led to development of polycystic kidneys and renal neoplasia. PLoS One 2008;3:e3581.

11. Hong $\mathrm{SB}, \mathrm{Oh} \mathrm{H}$, Valera VA, et al. Inactivation of the FLCN tumor suppressor gene induces TFE3 transcriptional activity by increasing its nuclear localization. PLoS One 2010;5:e15793.

12. Preston RS, Philp A, Claessens T, et al. Absence of the Birt-Hogg-Dube gene product is associated with increased hypoxia-inducible factor transcriptional activity and a loss of metabolic flexibility. Oncogene 2011;30:1159-1173.

13. Yang Y, Padilla-Nash HM, Vira MA, et al. The UOK 257 cell line: a novel model for studies of the human Birt-Hogg-Dube gene pathway. Cancer Genet Cytogenet 2008;180:100-109.

14. Pavlovich $\mathrm{CP}$, Grubb 3rd RL, Hurley $\mathrm{K}$, et al. Evaluation and management of renal tumors in the Birt-Hogg-Dube syndrome. J Urol 2005;173:1482-1486.

15. Benusiglio PR, Giraud S, Deveaux S, et al. Renal cell tumour characteristics in patients with the Birt-Hogg-Dube cancer susceptibility syndrome: a retrospective, multicentre study. Orphanet J Rare Dis 2014;9:163.

16. Iribe $\mathrm{Y}$, Kuroda $\mathrm{N}$, Nagashima $\mathrm{Y}$, et al. Immunohistochemical characterization of renal tumors in patients with Birt-Hogg-Dube syndrome. Pathol Int 2015;65:126-132.

17. Kunogi $M$, Kurihara $M$, Ikegami TS, et al. Clinical and genetic spectrum of Birt-Hogg-Dube syndrome patients in whom pneumothorax and/or multiple lung cysts are the presenting feature. J Med Genet 2010;47: 281-287.
18. Furuya M, Tanaka R, Koga S, et al. Pulmonary cysts of Birt-Hogg-Dube syndrome: a clinicopathologic and immunohistochemical study of 9 families. Am J Surg Pathol 2012;36:589-600.

19. Khoo SK, Kahnoski K, Sugimura J, et al. Inactivation of BHD in sporadic renal tumors. Cancer Res 2003;63:4583-4587.

20. Iribe $\mathrm{Y}$, Yao $\mathrm{M}$, Tanaka $\mathrm{R}$, et al. Genome-wide uniparental disomy and copy number variations in renal cell carcinomas associated with Birt-Hogg-Dube syndrome. Am J Pathol 2016;186: 337-346.

21. Brunelli $\mathrm{M}$, Eble $\mathrm{JN}$, Zhang $\mathrm{S}$, et al. Eosinophilic and classic chromophobe renal cell carcinomas have similar frequent losses of multiple chromosomes from among chromosomes 1, 2, 6, 10, and 17, and this pattern of genetic abnormality is not present in renal oncocytoma. Mod Pathol 2005;18:161-169.

22. Klomp JA, Petillo D, Niemi NM, et al. Birt-Hogg-Dube renal tumors are genetically distinct from other renal neoplasias and are associated with up-regulation of mitochondrial gene expression. BMC Med Genomics 2010;3:59.

23. Kato I, Iribe $Y$, Nagashima $Y$, et al. Fluorescent and chromogenic in situ hybridization of CEN17q as a potent useful diagnostic marker for Birt-Hogg-Dube syndrome-associated chromophobe renal cell carcinomas. Hum Pathol 2016;52:74-82.

24. Speicher MR, Schoell $B$, du Manoir $S$, et al. Specific loss of chromosomes 1, 2, 6, 10, 13, 17, and 21 in chromophobe renal cell carcinomas revealed by comparative genomic hybridization. Am J Pathol 1994;145:356-364. 